\title{
Levels and Trends of BMI, Obesity, and Overweight at National and Sub-national Levels in Iran from 1990 to 2016; A Comprehensive Pooled Analysis of Half a Million Individuals
}

\author{
Shirin Djalalinia, PhD ${ }^{1,2 *}$; Parinaz Mehdipour, MSC ${ }^{2 *}$; Bahram Mohajer, MD, MPH ${ }^{2 *}$; Farnam Mohebi, MD, MPH²; Bagher Larijani, MD $^{3}$;

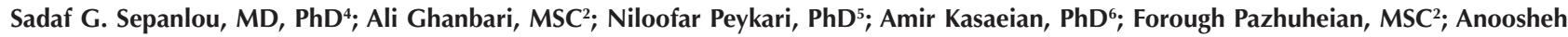 \\ Ghasemian, DDS²; Reza Malekzadeh, MD; Farshad Farzadfar, MD, MPH, DSC ${ }^{2,3^{*}}$, Collaborators"

\begin{abstract}
'Deputy of Research and Technology, Ministry of Health and Medical Education, Tehran, Iran
${ }^{2}$ Non-Communicable Diseases Research Center, Endocrinology and Metabolism Population Sciences Institute, Tehran University of Medical Sciences, Tehran, Iran

${ }^{3}$ Endocrinology and Metabolism Research Center, Endocrinology and Metabolism Clinical Sciences Institute, Tehran University of Medical Sciences, Tehran, Iran

${ }^{4}$ Digestive Diseases Research Center, Digestive Diseases Research Institute, Tehran University of Medical Sciences, Tehran, Iran ${ }^{5}$ Deputy for Education, Ministry of Health and Medical Education, Tehran, Iran

${ }^{6}$ Hematology, Oncology and Stem Cell Transplantation Research Center, Tehran University of Medical Sciences, Tehran, Iran
\end{abstract}

\begin{abstract}
Background: In developing countries like Iran, the burden of obesity increases through comorbid diseases. We estimated the mean body mass index (BMI) and prevalence of overweight/obesity by components of sex, age, province, and year in Iran from 1990 to 2016 .

Methods: Through a comprehensive systematic review, all relevant data sources pooled results with individual level national and sub-national population-based studies. Two stages of age-spatio-temporal modeling and Gaussian process regression were used to estimate mean BMI, followed by estimation of obesity and overweight prevalence through the crosswalk modeling. Results: In 2016, the age-standardized mean BMI was $27.9(27.2-28.7) \mathrm{kg} / \mathrm{m}^{2}$ in women and $25.9(25.2-26.5) \mathrm{kg} / \mathrm{m}^{2}$ in men. At the same time, the prevalence rates of overweight and obesity were $71.7 \%(67.9-75.8)$, and $36.8 \%(34.1-39.7)$ in females, and $57.1 \%(53.7-60.6)$, and $18.4 \%(16.9-20)$ in men. This shows a considerable increase from 1990 when the figures were respectively $24.4(23.3-25.5), 36.6 \%(32.2-41.5)$, and $8.2 \%$ (95\% UI: 6.9-9.7) in women, and 23.5 (22.5-24.5), 30\% (26.4-34), and 4.7\% (4.0-5.5) in men, with $66 \%$ attributed to population growth.

Conclusion: Considering the increasing trends of BMI, Sustainable Development Goals (SDGs) seem far out of reach. We need to call for action, aiming for both weight loss strategies and controlling the comorbidities that mediate high BMI risk. Keywords: Body mass index, Decomposition, Iran, Obesity, Sustainable development goals, Time trend Cite this article as: Djalalinia S, Mehdipour P, Mohajer B, Mohebi F, Larijani B, Sepanlou SG, et al. Levels and trends of BMI, obesity, and overweight at national and sub-national levels in iran from 1990 to 2016; a comprehensive pooled analysis of half a million individuals. Arch Iran Med. 2021;24(5):344-353. doi: 10.34172/aim.2021.51
\end{abstract}

Received: November 30, 2019, Accepted: December 16, 2020, ePublished: May 1, 2021

\section{Introduction}

High body mass index (BMI) and its increasing burden are important health issues in both developed and developing countries, rising as a global pandemic., ${ }^{1,2}$ The global age-standardized BMI average showed increasing trends from 22.1 (95\% credible interval 21.7-22.5) to 24.8 $(24.2-25.4) \mathrm{kg} / \mathrm{m}^{2}$ in females and from $21.7(21.3-22.1)$ to $24.5(24.3-24.6) \mathrm{kg} / \mathrm{m}^{2}$ in males, during the time period of 1975-2016. ${ }^{3}$ Based on the estimation of the global burden of diseases in 2015 , at least 4.0 million deaths $(7.1 \%$ of all deaths) and 120 million disability-adjusted life years (4.9\% of all disability-adjusted life-years) were attributed to increased weight. ${ }^{4}$ The huge rising burden of metabolic, cardiovascular, and musculoskeletal diseases associated with high $\mathrm{BMI}^{5}$ has placed this risk factor among 2025 Sustainable Development Goals (SDGs) for reduction of a $25 \%$ in premature mortality from non-communicable diseases (NCDs) by 2025, ${ }^{1,6,7}$ with halting the overweight and obesity rise as one of the goals that seems most unlikely to be achieved with the present trends. ${ }^{3}$

Contrary to individual level epidemiological studies, at the population level, the adjusted effect of high BMI on the mortality in high-income countries has been negligible. ${ }^{8,9}$ It has been suggested that pharmacological intervention

\footnotetext{
* These authors contributed equally to the work.

"Membership of the collaborators is provided in the acknowledgment

*Corresponding Author: Farshad Farzadfar, MD, MPH, DSc; Non-Communicable Diseases Research Center, Endocrinology and Metabolism Population Sciences Institute, Tehran University of Medical Sciences, Tehran, Iran. Second Floor, No.10, Jalal Al-e-Ahmad Highway, Tehran, Iran. Tel: +98-21-88631293; 1411713137; Email: f-farzadfar@tums.ac.ir
} 
could effectively modify the mediators of the impact of BMI on cardiovascular diseases, namely blood pressure, serum cholesterol, and diabetes. ${ }^{1}$ In developing countries like Iran, where besides the suboptimal awareness of general population, access, equity and efficiency of healthcare system regarding these mediators may be modest, ${ }^{10-12}$ the future burden of high BMI may be devastating.

Considering the global commitments on the reduction of the risk and by the obligations enshrined in a national action plan documents' for non-communicable diseases prevention and control in Iran, ${ }^{13}$ policy makers and health domains managers need reliable evidence on the levels, trends, and distribution of NCDs risk factors. ${ }^{6,7}$ In a recent NCD-RisC study, it was estimated that the average BMI in Iran in 2016 has reached $25.4 \mathrm{~kg} / \mathrm{m}^{2}$ and $27.3 \mathrm{~kg} / \mathrm{m}^{2}$ in males and females, respectively. ${ }^{3}$ Despite the possible catastrophic future of obesity-attributed NCDs, and the requirement for a precise estimate of the BMI burden for effective policy planning, the published estimates of the Iranian population are scattered and mostly limited to subgroups of the population. ${ }^{14-16}$ Moreover, trend estimations mainly lack report of mean BMI and its distribution, ${ }^{17}$ are extracted from model-driven approaches ${ }^{18}$ and do not consider subnational distribution. ${ }^{3}$

To face the needs and by benefitting from all available data, the present investigation aims to estimate the trends of mean BMI as well as overweight and obesity prevalence in the Iranian adult population, considering sex, age, and geographical distribution, from 1990 to 2016, at both national and sub-national levels.

\section{Materials and Methods}

We estimated trends of mean BMI and prevalence of overweight and obesity according to sex, and 5-year age groups for all 31 provinces of Iran, in the adult population aged 25 to 85 years, during 1990-2016. BMI was defined as weight $(\mathrm{kg})$ divided by height squared $\left(\mathrm{m}^{2}\right)$. For practical analysis, overweight and obesity were considered as BMI $\geq 25 \mathrm{~kg} / \mathrm{m}^{2}$ and $\geq 30 \mathrm{~kg} / \mathrm{m}^{2}$, respectively. ${ }^{1,19}$ We run a systematic review for published and unpublished related data in international and national Iranian literature, as well as data of population-based cohort studies, following the standard protocols (Supplementary file 1). ${ }^{20}$

Through fitting a proportional linear mixed effect model, the log mean of BMI was extracted from components of sex (male/female), age-specific (13 age groups from 25 to 85 years categorized as 5 -year age intervals), geographical areas (31 provinces), and study years (from 1990 to 2016). In order to follow a normal distribution, the modelling approach followed a log transformation on BMI.

Years of schooling, wealth index, and urbanization were included as fixed-effects covariates of the model and province was considered as a random effect of the linear mixed effect model. This model was reiterated separately for women and men. We standardized all the estimates with the Iranian national census population of the last year which was 2016. We aimed to consider the variation between sex-age-year-province combinations by socioeconomic status measures including years of schooling, wealth index, and urbanization. These variables helped us to improve the final national and sub-national estimates as they have high correlation with healthrelated measures and risk factors. Years of schooling were measured according to the level of education attainment for each person extracted from the Statistical Center of Iran (Household and Income Survey). This variable varied from 0 to 25 years. We calculated wealth index based on the Household and Income Survey data for each household. We performed a principal component analysis over all the assets and income of each household. Urbanization was calculated according to the population size living in the urban areas over total population size for each age-sexyear-province combination ranging from $0 \%$ to $100 \%$. It is worth mentioning that we transformed each variable to logarithms scale to obtain normal distribution.

The extra variations in age, location, and time were measured by the age-spatio-temporal model (detailed explanation of the model is provided in Supplementary file 1), fitted based on the residuals extracted from the linear mixed effect model and residuals were smoothed accordingly. Through the modelling, by smoothing the residuals, we adopted strengths of year, age groups and provinces. To do this, we calculated adjacency matrices over age, province and year. Regarding these matrices, the adjacent provinces, years and similar age groups will be more consistent with each other. Since this model included the neighboring provinces data and benefitted from nearby time points and ages, it can improve BMI estimates compared to the linear mixed effect model.

In the final step, in order to unify all data sources and estimate their uncertainty intervals over time, we used Gaussian process regression (GPR). ${ }^{21-23}$ To address the various population sizes of different studies, we considered the sample size of each study as one of the variance components in the GPR model. The variance in GPR model includes two parts for taking into account the variance in each study and between studies. The sampling variance shows the variation resulting from the sampling and small numbers of study with the inverse probability of studies' samples. The measurements' uncertainty of non-sampling error was considered for each type of data sources. These parameters were estimated when source tends disagreed with the other sources. The more a source conflicts with other sources, the higher the variance parameter will be and the higher uncertain the measurements. In Gaussian process regression, as the covariance function, the Matern function, determined the amount of borrowing neighborhood correlation in timespan.

Three parameters controlled the correlation and smoothness of estimated time trends. We made all calculations using a hyperparameter in the Bayesian 
model. The details of these model are described in Supplementary file 1.

Using crosswalk models, the prevalence of overweight and obesity was calculated from BMI mean estimates; a regression model in which the extracted BMI mean of GPR model was included as an independent variable (Supplementary file 1).

Uncertainty was estimated for both cross-sectional and over-time uncertainties. This Bayesian GPR model uses sampling and also non-sampling errors to capture all the variation in each data source and between data sources. This is the sum of inverse variance of each data source for sampling error and inverse Gamma distribution for non-sampling errors. To draw samples $(n=5000)$ from the posterior distributions of model parameters for estimating the prevalence, the Markov Chain Monte Carlo method combined the empirical data, model priors and the median. Based on this approach, the 2.5 percentile and 97.5 percentile of distributions were considered as point estimates of lower and upper UIs, respectively.

We used a two-phase sensitivity analysis to assess the validity of estimates of BMI for each age-sex-location combination. First, we randomly masked $10 \%$ of data points and repeated all the analyzing process for the rest of the data. To evaluate the performance of in-sample validity for metrics of root mean square error (RMSE), Root median square error, median relative error, and mean relative error of prediction models were used. Median relative error and mean relative error were estimated as the fraction of mean or the fraction of median absolute deviation of observations. For BMI, $96 \%$ of data hold in $90 \%$ prediction of model and its uncertainty.

After that, calculation followed for the proportion of data points in the masked data set that fell within the 95\% uncertainty interval (UI) of $90 \%$ withheld data.

To estimate the contribution of increased prevalence, population growth, and change in age structure, in the changed number of population with obesity and overweight during 1990-2016, we used decomposition analysis. To estimate the contribution of each of them, we estimated the percent change in population of 1990 attributed to specific factor while considering the two remaining factors constant during these years.

\section{Results}

\section{Systematic Review}

From the total 8013 searched records, 5086 papers remained for refinement after exclusion of duplications. Through screening of studies by two separate authors and judgment of a third author in case of disagreement, a total of 172 studies were assessed in the data collection process. From 172 studies, 129 were excluded due to limitations in presenting the required data or unacceptable quality of reported results according to the study criteria (Supplementary file 1), leading to the final inclusion of 43 studies in the data collection. We also pooled the aggregated data of seven population-based cohort studies, individual-level data of seven rounds of national surveys of NCD risk factors $(2005,2006,2007,2008,2009,2011$, and 2016 rounds of STEPwise approach to surveillance studies or STEPs), and one iteration of National Health Survey (NHS) in 2000. At last, the systematic search resulted in 4,633 total data points for more than 471,500 individuals aged 25 to over 85 years, from the populationbased surveys in Iran. The results of the systematic search steps and data sources for each province and year are shown in the heat map in Supplementary file 1 (Figure S1, Figure S2 and Table S1).

\section{Sex-Specific Trends}

Between 1990 and 2016, in the female population, agestandardized average BMI at national level increased from $24.38(23.3-25.5) \mathrm{kg} / \mathrm{m}^{2}$ to $27.9(27.2-28.7) \mathrm{kg} / \mathrm{m}^{2}$. This is indicative of $14.6 \%$ (6.6-23.1) change with average of 0.14 $\mathrm{kg} / \mathrm{m}^{2}$ increase in BMI each year. In contrast, the described trend in BMI was modest in the male population during the 27 years; an increase from $23.5(22.5-24.5) \mathrm{kg} / \mathrm{m}^{2}$ to $25.9(25.2-26.5) \mathrm{kg} / \mathrm{m}^{2}$ with percent change of $10.2 \%$ (2.918.1) (Figure 1 and Figure S3) and an average $0.09 \mathrm{~kg} / \mathrm{m}^{2}$ increase in BMI each year. The growing trend of BMI from 1990 to 2016 has resulted in an increased prevalence of both the population with obesity and the population with overweight. The age-standardized overweight prevalence considerably increased from $36.6 \%(32.2-41.5)$ in 1990 to $71.7 \%(67.9-75.8)$ in 2016 in females, and following a similar trend in males, rose from $30 \%(26.4-34)$ to $57.1 \%$ (53.7-60.6) (Figure S3). This equals to a $96 \%(64 \%-135 \%)$ and $90 \%(58 \%-129 \%)$ change in the Iranian female and male population with obesity, respectively. From 1990 to 2016, the number of individuals with overweight changed from 6.4 (5.6-7.3) million (3.4 million [53\%] females) to 31.7 (29.9-33.6) million (17.5 million [55\%] females) (Figure 2). These findings were almost homogeneous across provinces.

The age-standardized prevalence of obesity in females increased from $8.2 \%$ (95\% UI: 6.9-9.7) in 1990 to $36.8 \%$ (34.1-39.7) in 2016, while an increase from $4.7 \%$ (4.0$5.5)$ to $18.4 \%(16.9-20)$ was observed in males (Figure 1 and Figure S3). The increase is indicative of a widening sex gap trend, five times bigger in 2016 compared to 1990. The prevalence of obesity almost tripled in both sexes from 1990 to 2016; 348\% (251\%-471\%) and 292\% $(206 \%-401 \%)$ change in females and males, respectively. Nationally, the number of the Iranian population with obesity increased from 1.2 (95\% UI 1.0-1.4) million (0.76 million [63\%] female) in 1990 to 13.6 (12.6-14.7) million (9.0 million [66\%] female) in 2016 (Figure 2).

Sex-specific crosswalk models between BMI overweight prevalence and BMI obesity prevalence were indicative of similar slopes for sexes considering overweight prevalence but a greater slope in the female population compared to males, regarding obesity prevalence (Figure 3, Figures S4-S6).

When comparing BMI distribution in female and male 
National age-standardized mean BMl
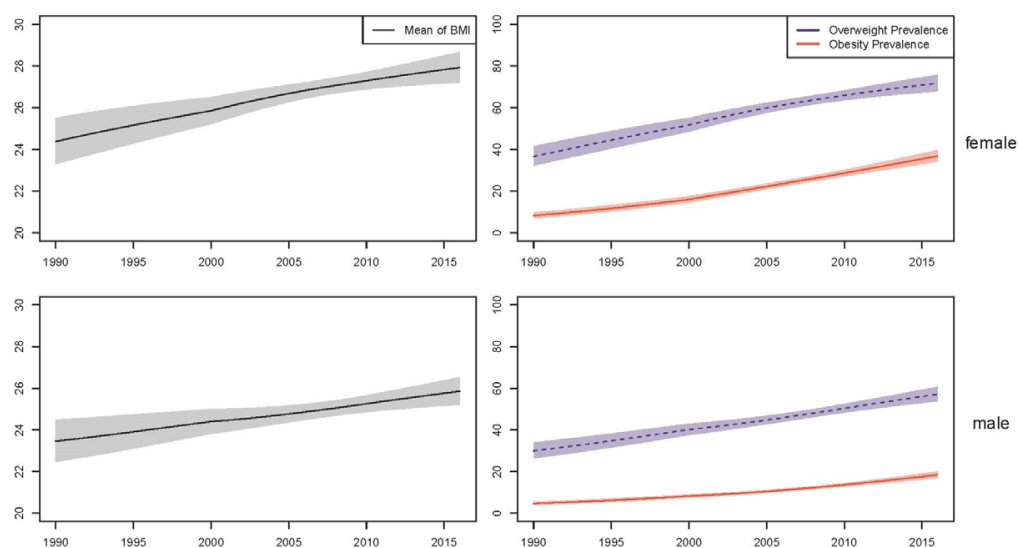

Figure 1. Trends in National Age-Standardized Mean BMI, Overweight and Obesity Prevalence by Sex in People Aged 25 Years and Older From 1990 to 2016. The lines show the posterior mean estimates and the shaded areas show the $95 \%$ UI.
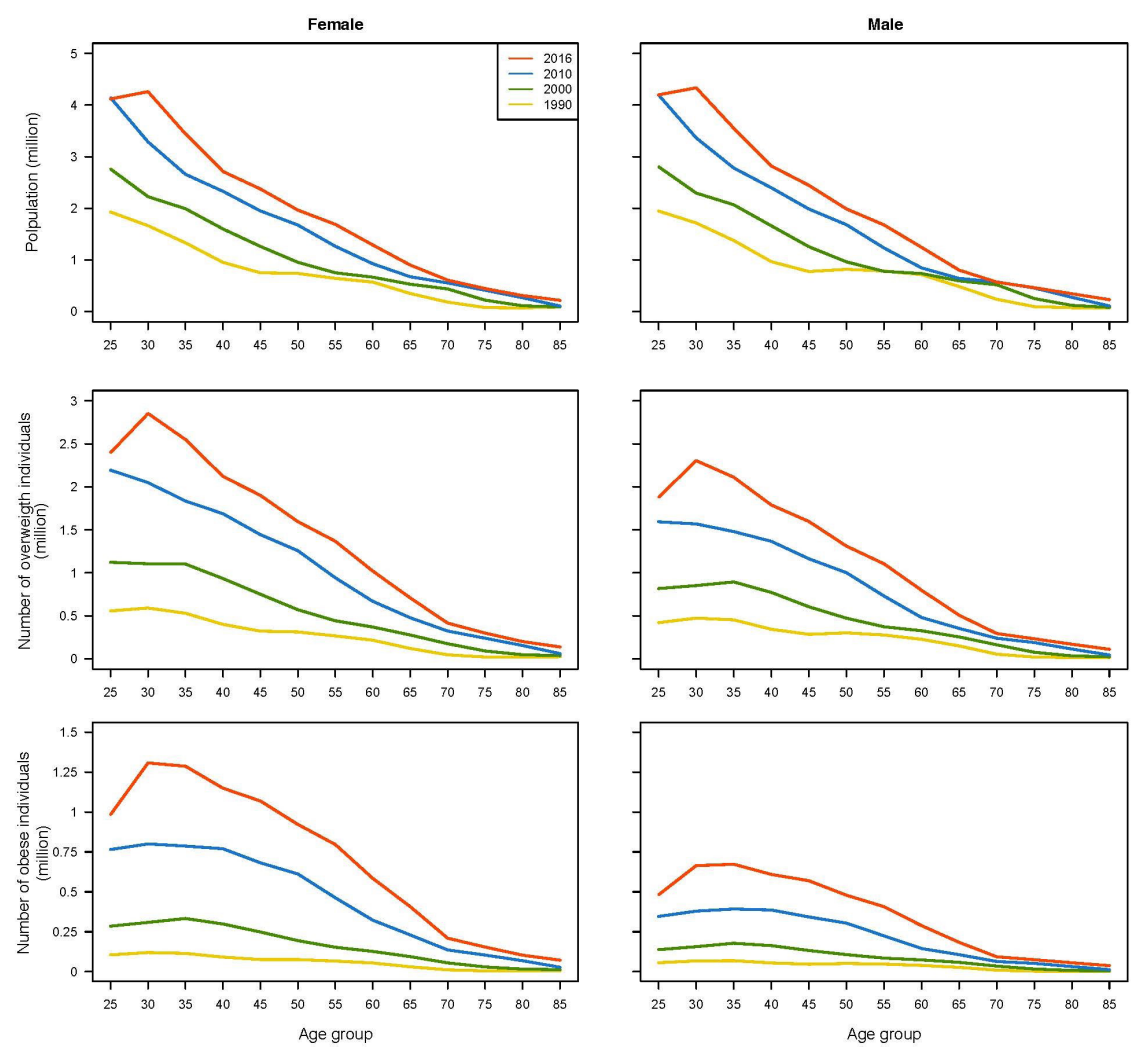

Figure 2. The National Population, Number of Obese and Overweight Individuals by Sex and by Age Groups in People Aged 25 Years and Older.

populations, between 1990 and 2016 (Figure 4), despite the right shift (increase) in the average BMI of both male and female populations, two peaks can be seen in the distribution of BMI in both sexes and during this time period. However, this diverging growth of the BMI is more highlighted in the female population and more exaggerated in 2016, leading to a higher standard deviation. When considering the age groups separately (Figures S6-S8), age groups ranging from 35 to 65 years had the highest BMI in 1990 in both sexes, but most of the distribution were below the BMI of 25. In 2016, the distribution of all these age categories shifted to the right, falling in the overweight and obese categories. Age categories at extremities of +75 and 25-35 were less affected by BMI increase in both sexes, distributed mostly around the normal-overweight BMI border. The very same diverging pattern in BMI distribution could be seen across socio-economic levels assessed by quantiles of wealth index; the richest quartiles showed a faster pace in BMI increase during these years (Figure S9).

Throughout all years, prevalence of obesity and overweight for age groups of 25 to 70 -year-old showed 

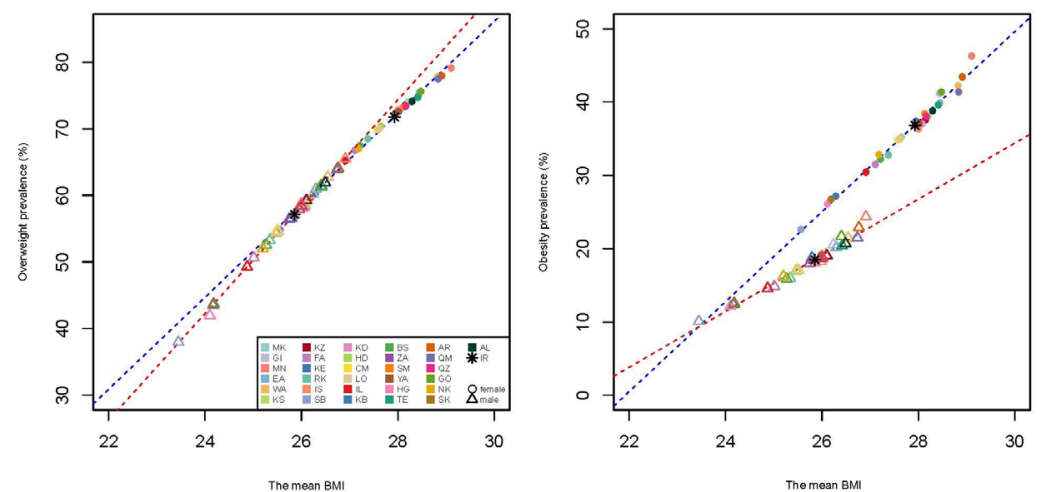

Figure 3. Relation Between Age-Standardized Obesity Prevalence and Overweight Prevalence with Mean BMI in Individuals Aged 25 Years and Older.

a dome-shaped pattern followed by a plateau in the population groups of both sexes aged more than 70 in all years. We observed the highest prevalence of obesity and overweight among the 55-60-year-old age group (Figure S10).

Generally, in 2016, the highest average BMI, prevalence
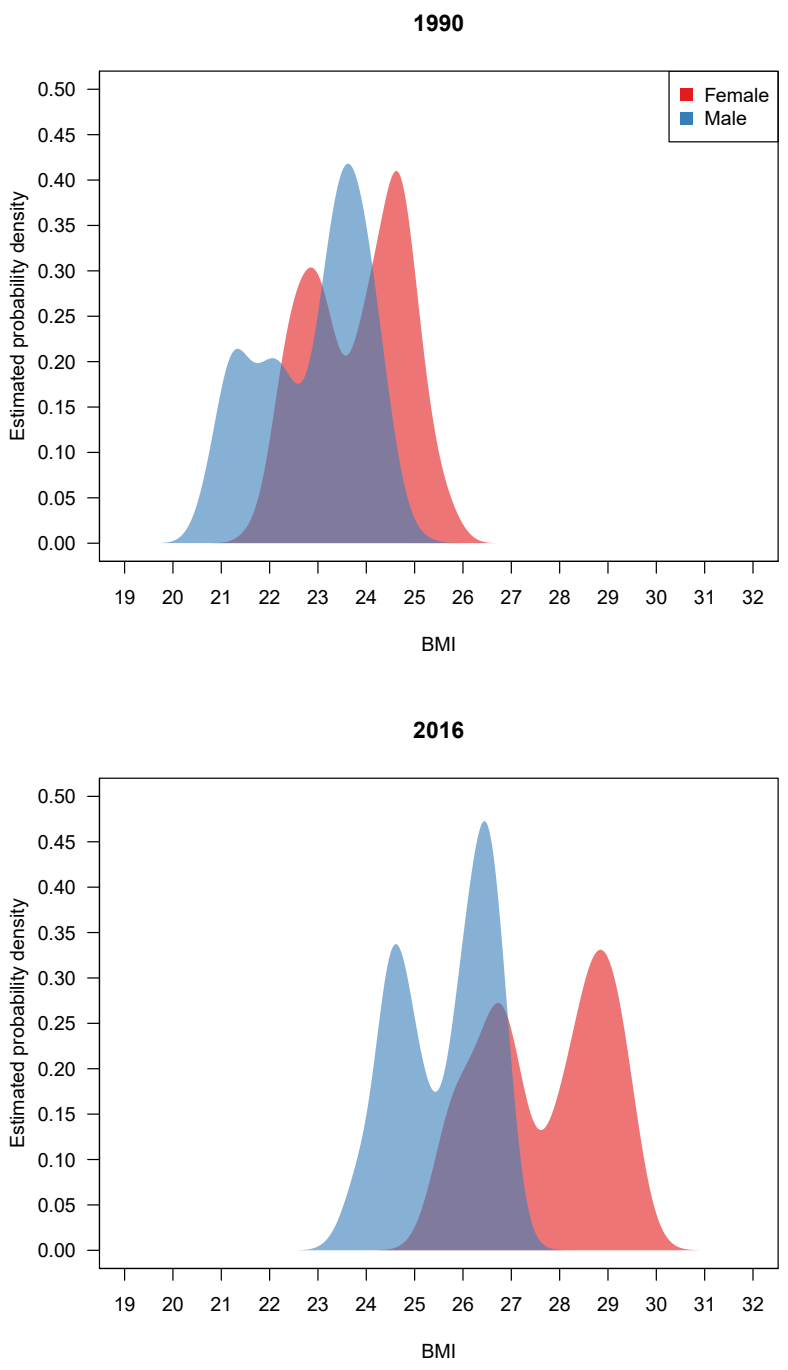

Figure 4. Distribution of Mean BMI by Sex in People Aged 25 Years and Older in 1990 and 2016. of overweight and obesity were seen in the northern and western provinces and decreased when moving towards southern and eastern regions (Figure 5). Throughout most years, these numbers were on average highest in Mazandaran and lowest in Sistan and Baluchistan provinces in both sexes. In females, the mean agestandardized BMI in 1990 ranged from 22.48 (20.5-24.5) $\mathrm{kg} / \mathrm{m}^{2}$ in Sistan and Baluchistan to $25.3(24.3-26.3) \mathrm{kg} /$ $\mathrm{m}^{2}$ in Alborz, and in 2016, from $25.5(24.4-26.6) \mathrm{kg} / \mathrm{m}^{2}$ in Sistan and Baluchistan to $29.1(28.1-30.1) \mathrm{kg} / \mathrm{m}^{2}$ in Mazandaran. In males, in 1990, the mean BMI ranged from $21.3(19.4-23.2) \mathrm{kg} / \mathrm{m}^{2}$ in Sistan and Baluchistan to $24.4(23.4-25.3) \mathrm{kg} / \mathrm{m}^{2}$ in Tehran, and in 2016 from 23.4 $(22.4-24.5) \mathrm{kg} / \mathrm{m}^{2}$ again in Sistan and Baluchistan to 26.91 (25.9-27.9) kg/m² in Qazvin (Figure S11 and Table S3). In terms of percent change in the mean BMI of provinces from 1990 to 2016, subnational numbers ranged from $11.7 \%$ (5.0-18.7) in Alborz to $17.5 \%$ (8.6-27.1) in West Azerbaijan in females. In males, Tehran with 8.3\% (1.915.2) change was considerably more stable compared to other provinces while North Khorasan had the highest variation of $12.6 \%(6.9-18.4)$ throughout these years (Table S3). Overall, provinces at both extremes of the lowest and highest numbers for mean BMI, prevalence of obesity and overweight showed greater percent change, while provinces placed near national average had moderate percentage changes (Figure S12).

Decomposition Analysis

Decomposition analysis revealed that $65.7 \%$ of the increase in number of adults with overweight was attributed to population growth, 33\% due to increased age-standardized prevalence, and $1 \%$ due to change in population age structure. For obesity percent change, the estimates were $75.4 \%, 24 \%$, and $0.6 \%$, respectively (Figure S13).

\section{Sensitivity Analysis}

Using sensitivity analysis, we calculated the proportion of data points in our masked data set that was located in the $95 \%$ UI of our $90 \%$ withheld BMI data. The results showed that $96 \%$ of $10 \%$ withheld data to hold in $90 \%$ prediction 


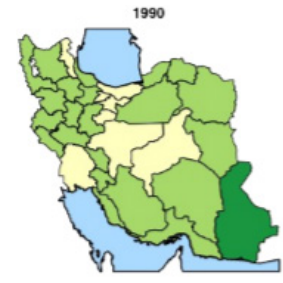

2010

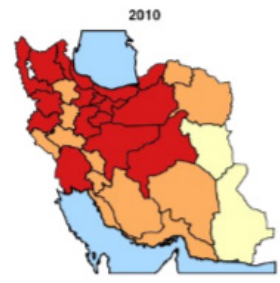

a. Female

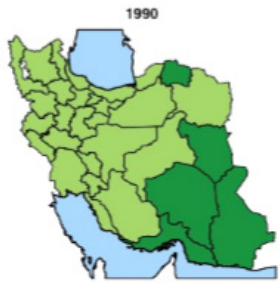

2010

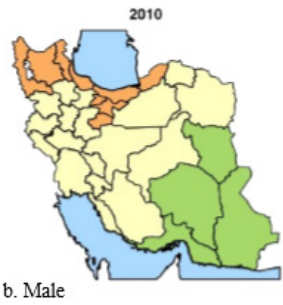

. Male

Figure 5. Age-Standardized Prevalence of Overweight and Obesity by Province and by Sex in People Aged 25 Years and Older in 1990, 2000, 2010 and 2016.

of model and its UI, indicative of accuracy of estimations.

\section{Discussion}

We estimated the trends of mean BMI as well as overweight and obesity prevalence in the Iranian adult population, by sex, age, and geographical distribution, from 1990 to 2016 . We found a considerable increase in the mean BMI, age-standardized prevalence of overweight population and more importantly a three-fold increase in the prevalence of obesity in Iran. With population growth as the primary reason, and increased mean BMI as the second contributor, the number of people with obesity has increased exponentially by nearly 11 -folds during these years. Consistent with global trends, ${ }^{3,24-26}$ the female population has been more severely affected throughout these decades, showing numbers twice in size in both percent change and cross-sectional endpoints in 2016. This is indicative of a diverging growth pattern between sexes. Average BMI and prevalence of obesity and overweight increased steadily with age, with the 5060 age groups constantly showing the highest mean BMI. Moreover, the dome-shaped pattern of obesity prevalence for the age groups showed a greater curve in the recent years, highlighting the more severe increase of obesity in the middle-aged categories, the population susceptible for other comorbid NCDs. ${ }^{27}$

Our results show that by continuing the same trends, the Iranian population would be far away from reaching the 2025 SDG goals in halting the overweight/obesity rise. ${ }^{6}$ In Iran, similar to other developing countries, increased BMI can be primarily attributed to lifestyle changes which have occurred in the recent decades related to rapid socioeconomic development, more Western lifestyle, ${ }^{28}$ insufficient physical activity, ${ }^{29}$ and urbanization accompanied by rural-urban migration. ${ }^{4,30}$ However, a complex combination of leading causes of biological and non-biological components can also contribute, where age, sex, race/ethnicity, and socioeconomic status interact with lifestyle habits. ${ }^{26,31,32}$ Accepting the clinical importance of the underlying pathophysiology, which is mainly shared between populations and can be elucidated through experimental and clinical studies, the distribution of increased BMI in Iran needs great attention; observing the highest average BMI, prevalence of obesity and overweight in the northern and western provinces alongside a slight decrease when moving towards southern and eastern regions.

It has been shown that the standard deviation of BMI distribution, as a proposed measure of equity, has been increased during the past three decades in the world, ${ }^{3}$ similar to what we observed in the Iranian population. The increased SD, instead of being a result of increased deviation from the mean, was caused by the diversification of the population into two sub-populations, each concentrated around their new mean. Despite the diffuse increase in the mean BMI of the population, the slope is much greater in those aged 35-65 years, females, of middle to high socio-economic status, and located in the northern provinces (not only the highest prevalence of obesity and overweight, but also the highest percent change during these years). Notably, the lowest physical activity level was seen in the very same population subgroups in Iran. ${ }^{33}$ To reach the defined goals in a diverging population, interventions need to be primarily designed for the subgroups that showed more rapid weight gain.

Several studies conducted on obesity trends in Iran and the suggested trends are mainly consistent with what we have found. ${ }^{1,3,17,19,24}$ The Global Burden of Diseases (GBD) study showed obesity prevalence in Iranian adult females and males ( $\geq 20$ years) at $29.3 \%$ and $13.6 \%$, respectively, lower than our estimates. ${ }^{18}$ In the latest study of the NCDRisC group, between 1975 and 2016, the age-standardized prevalence of obesity in females increased from $10.9 \%$ to $33.5 \%$ (mean BMI change of 23.3 to $27.3 \mathrm{~kg} / \mathrm{m}^{2}$ ) and in males, changed from $3.0 \%$ to $20.0 \%$ (mean BMI change of 21.8 to $\left.25.4 \mathrm{~kg} / \mathrm{m}^{2}\right){ }^{3}$ This is almost similar to our results, although reporting a milder between-sex gap. Compared to previous studies, we used subnational data points for covariates in the model, and therefore, we believe our 
estimates may present a more realistic picture of BMI in the Iranian population.

Looking at the distribution of BMI and obesity in the world, it seems that variance is more described by the region rather than the economic status. ${ }^{3}$ Iran has a prevalence of obesity and means of BMI lower than what is seen in most Middle East - North Africa countries such as Saudi Arabia, Turkey, and Iraq, but higher than the eastern neighbors including Turkmenistan, Afghanistan, and Pakistan. ${ }^{3}$ It is interesting to mention that the same decreasing BMI spectrum from western Middle Eastern - North African countries continuing to east Asia, can be seen as a geographical trend in western provinces towards eastern ones in Iran (Figure 6). This emphasizes the emergence of an internationally synchronized look to policy making concerning obesity with respect to the incompetency of past national interventions to halt BMI increase in most of the countries. ${ }^{3}$

Several points strengthened the present study. It is the first time that sex-specific subnational trends for BMI and prevalence and obesity are estimated in Iran. We conducted a comprehensive up-to-date systematic search that included all published and unpublished quality data sources empowering our models with quite a high number of data-points. Moreover, we benefitted from age-spatio-temporal and GPR models, inclusion of highresolution subnational level data (such as wealth index) as covariates in the model, calculation of uncertainty intervals, sensitivity analysis confirming our estimations, and decomposition analysis showing the contributing factors.

However, we faced limitations. First, we encountered scarcity and low quality of data in published studies in several age-sex-location groups. We tried to address this limitation by quality-weighting of the studies, inclusion of population-based national cohorts and individual level data of national surveys, and inclusion of subnational covariates in the advanced statistical models. Second, for estimating the prevalence of obesity and overweight, we used a crosswalk model on estimated mean BMI which may have resulted in imprecise estimation in some provinces due to the high variance of BMI distribution across the country. However, the same method has been widely used in previous studies and approved as one of the best substitutes. ${ }^{1,3,24,34}$ Finally, age categorization was inconsistent among several included studies. We tried to define age groups based on the studies with the highest weight in the analysis.

Rapid BMI increase during the past years in Iran has occurred lately as an acute complication in the health system. Only recently in 2016, a comprehensive national action plan for NCDs was developed in Iran, aiming to control overweight and obesity as one of the SDGs ${ }^{13,35}$ and it is still too early to evaluate the outcomes. According to our findings, high BMI is unequally distributed throughout the country, with considerably more rapid increase in a subgroup of population who are at great risk

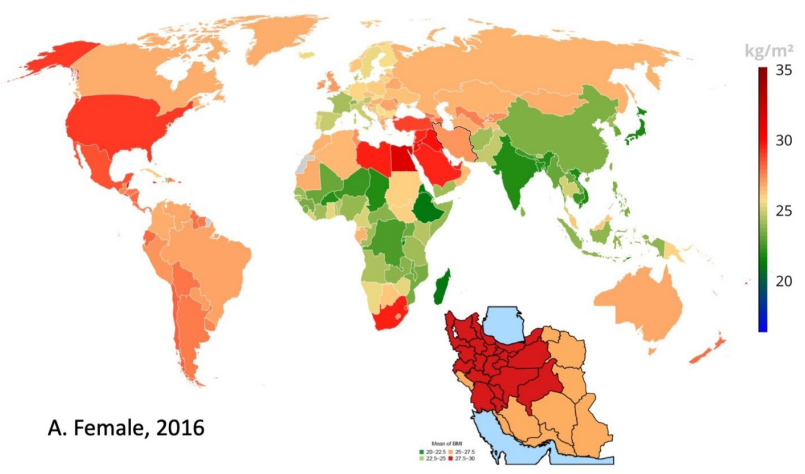

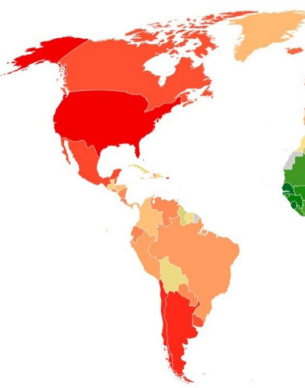

B. Male, 2016
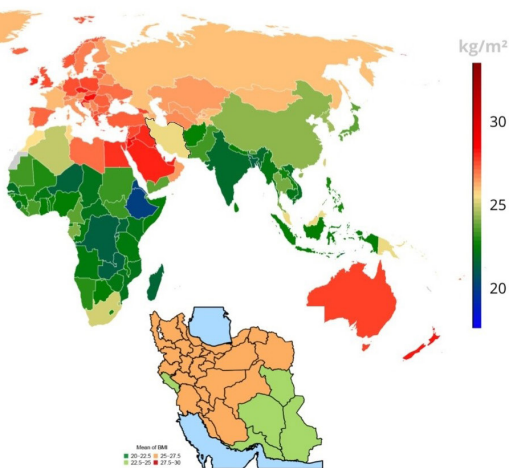

Figure 6. Comparison of Age-Standardized Mean BMI by Sex in People Aged 25 Years and Older in Iran and All Other Countries of the World in 2016. Data from NCD Risk Factor Collaboration (NCD-RisC) group presented with permission.

of other comorbid NCDs associated with obesity. It has been shown that increased BMI is associated with higher mortality in developing countries, where inefficiency or inequality in health systems may lead to unsuccessful identification or treatment of dyslipidemia, hypertension, and diabetes as effect modifiers of obesity. ${ }^{3,8,9}$ With aging of the population in Iran, ${ }^{36}$ the very same middle-aged population with the highest BMI may develop comorbid NCDs, leaving a massive burden on the health system. Interventions targeting the lifestyle such as modifications of nutritional habits, promoting physical activity, and behavioral therapy are among the main potential routes for weight management. ${ }^{2,37,38}$ Considering that the distribution of high BMI mostly mimics insufficient physical activity in age, sex, and socio-economic status, ${ }^{29}$ behavioral intervention aiming at physical activity promotion may be most efficient.

Accepting the fact that inter-sectoral policies have to be made for behavioral interventions to promote weight loss, the global trend questions the population-level effectiveness of such policies in developing countries similar to Iran and increasing trends are projected to continue. ${ }^{3}$ Thus, for the most effective interventions, we suggest prioritizing the subgroup of population who have more rapidly gained weight during the past decades: the middle-aged, females, those in the middle to high socioeconomic status, throughout the northern and western part of the countries. Controlling the effect mediators and 
possible co-morbidities associated with increased BMI, such as hypertension and diabetes, may be more attainable and more cost-effective. ${ }^{39,40}$ Considering the complexity of background and increasing complications of obesity, a scientific view of the problem needs the required information for parallel individual and population interventions, which would be designed for specific target groups. ${ }^{31,41-44}$ As a noticeable suggestion, individual and social behaviors that are related to weight gain should be more subject to research studies. ${ }^{45}$

In this nationally representative study of adults in Iran, the age-standardized prevalence rates of obesity and average BMI in 2016 were $40.4 \%$ and $27.9 \%$ in the female and $35.0 \%$ and $25.9 \%$ in the male population - a tripled prevalence of obesity compared to 1990 . The tensity of the burden is more prominent in the middle-aged, females, and in northern and western provinces. The female and middle-aged population not only had higher numbers for all measures, but also showed a more rapid increasing pace during these years, resulting in a bifurcating growth pattern of BMI distribution between the two sexes. Continuing the present pattern makes SDGs impossible to achieve. With future clinical studies aiming to elucidate the exact contributing factors of obesity in the Iranian population, agile policies should be made for the most affected, susceptible, and relatively easily affectable subgroups to reach the SDGs.

\section{Authors' Contribution}

FF, SD and PM developed the main design of manuscript. All co-authors had contribution and participated in the revision of the manuscript.

\section{Conflict of Interest Disclosures}

The authors declare that they have no conflict of interest.

\section{Role of Funding Source}

The funding sources of the study had no role in study design, data collection, data analysis, data interpretation, or writing of the report. FF, as the corresponding author, had full access to the data in the study and had final responsibility for the decision to submit for publication.

\section{Ethical Statement}

Study did not directly include any human participants and was ethically approved by institutional research board committee of Iran Ministry of Health and Medical Education (MoHME) (IR. NIMAD.REC.1397.513).

\section{Funding}

The grant of the study comes from the Ministry of Health and Medical Education of Islamic Republic of Iran and National Institute for Health Research. This research was supported by NIMAD (The National Institute for Medical Research Development)(Grant No. 973048).

\section{Acknowledgements}

The authors would like to express their appreciation for partnership of deputy for health and deputy for research and technology of the Ministry of Health and Medical Education, Islamic Republic of Iran's. We would also like to express thanks to all of participants and scientific and executive partners, who made this experience possible.

The authors would like to thank the extended staff of all the collaborators involved in the project, consist of the following members and their affiliations:

Fereydoun Azizi, Endocrine Research Center, Research Institute for Endocrine Sciences, Shahid Beheshti University of Medical Sciences, Tehran, Iran. Davood Khalili, Prevention of Metabolic Disorders Research Center, Research Institute for Endocrine Sciences, Shahid Beheshti University of Medical Sciences, Tehran, Iran. Masoud Khoshnia, Golestan Research Center of Gastroenterology and Hepatology, Golestan University of Medical Sciences, Gorgan, Iran. Jalil Kouhpayehzadeh, Center for Educational Research in Medical Sciences (CERMS), Iran University of Medical Sciences, Tehran, Iran. Nizal Sarrafzadegan, Isfahan Cardiovascular Research Center, Cardiovascular Research Institute, Isfahan University of Medical Sciences, Isfahan, Iran. Shahin Merat, Liver and Pancreaticobiliary Disease Research Center, Digestive Diseases Research Institute, Tehran University of Medical Sciences, Tehran, Iran. Ali Mirzazadeh, Department of Epidemiology and Biostatistics, University of California San Francisco, San Francisco, United States. Kazem Mohammad, Department of Biostatistics and Epidemiology, School of Public Health, Tehran University of Medical Sciences, Tehran, Iran. Noushin Mohammadifard, Hypertension Research Center, Cardiovascular Research Institute, Isfahan University of Medical Sciences, Isfahan, Iran. Iraj Nabipour, The Persian Gulf Marine Biotechnology Research Center, The Persian Gulf Biomedical Sciences Research Institute, Bushehr University of Medical Sciences, Bushehr, Iran. Mahboubeh Parsaeian, Department of Biostatistics and Epidemiology, School of Public Health, Tehran University of Medical Sciences, Tehran, Iran. Akram Pourshams, Digestive Diseases Research Center, Digestive Diseases Research Institute, Tehran University of Medical Sciences, Tehran, Iran. Hossein Poustchi, Liver and Pancreaticobiliary Disease Research Center, Digestive Diseases Research Institute, Tehran University of Medical Sciences, Tehran, Iran

\section{Supplementary Materials}

Supplementary file 1 contains Figures S1-S13 and Tables S1-S3.

\section{References}

1. Collaboration NRF. Trends in adult body-mass index in 200 countries from 1975 to 2014: a pooled analysis of 1698 population-based measurement studies with 19. 2 million participants. Lancet. 2016;387(10026):1377-1396. doi: 10.1016/S0140-6736(16)30054-X.

2. Djalalinia S, Qorbani M, Peykari N, Kelishadi R. Health impacts of Obesity. Pak J Med Sci. 2015;31(1):239-42. doi:10.12669/pjms.311.7033.

3. NCD Risk Factor Collaboration (NCD-RisC). Worldwide trends in body-mass index, underweight, overweight, and obesity from 1975 to 2016: A pooled analysis of 2416 population-based measurement studies in 128.9 million children, adolescents, and adults. The Lancet. 2017;390 (10113):2627-42.

4. Afshin A, Forouzanfar MH, Reitsma MB, Sur P, Estep K, Lee A, et al. Health effects of overweight and obesity in 195 countries over 25 years. N Engl J Med. 2017;377(1):13-27. doi: 10.1056/NEJMoa1614362.

5. Prospective Studies C. Body-mass index and cause-specific mortality in 900000 adults: collaborative analyses of 57 
prospective studies. Lancet. 2009;373(9669):1083-96. doi: 10.1016/S0140-6736(09)60318-4.

6. WHO. Global action plan for the prevention and control of noncommunicable diseases 2013-2020. Geneva, Switzerland: World Health Organization; 2013.

7. Kontis V, Mathers CD, Rehm J, Stevens GA, Shield KD, Bonita $\mathrm{R}$, et al. Contribution of six risk factors to achieving the $25 \times 25$ non-communicable disease mortality reduction target: a modelling study. Lancet. 2014;384(9941):427-37. doi: 10.1016/S0140-6736(14)60616-4.

8. Di Cesare M, Bennett JE, Best N, Stevens GA, Danaei G, Ezzati M. The contributions of risk factor trends to cardiometabolic mortality decline in 26 industrialized countries. Int J Epidemiol. 2013;42(3):838-48. doi: 10.1093/ ije/dyt063.

9. Ezzati M, Obermeyer Z, Tzoulaki I, Mayosi BM, Elliott $\mathrm{P}$, Leon DA. Contributions of risk factors and medical care to cardiovascular mortality trends. Nat Rev Cardiol. 2015;12(9):508-30. doi: 10.1038/nrcardio.2015.82.

10. Zare H, Trujillo AJ, Driessen J, Ghasemi M, Gallego G. Health inequalities and development plans in Iran; an analysis of the past three decades (1984-2010). Int J Equity Health. 2014;13:42. doi: 10.1186/1475-9276-13-42.

11. Moradi-Lakeh M, Bijari B, Namiranian N, Olyaeemanesh AR, Khosravi A. Geographical disparities in child mortality in the rural areas of Iran: 16-years trend. J Epidemiol Community Health. 2013;67(4):346-9. doi: 10.1136/jech2012-201511.

12. Lankarani KB, Alavian SM, Peymani P. Health in the Islamic Republic of Iran, challenges and progresses. Med J Islam Repub Iran. 2013;27(1):42-9.

13. Peykari N, Hashemi H, Dinarvand R, Haji-Aghajani M, Malekzadeh R, Sadrolsadat A, et al. National action plan for non-communicable diseases prevention and control in Iran; a response to emerging epidemic. J Diabetes Metab Disord. 2017;16:3. doi: 10.1186/s40200-017-0288-4.

14. Pishdad GR. Overweight and obesity in adults aged 20-74 in southern Iran. Int J Obes Relat Metab Disord. 1996;20(10):963-5.

15. Najafipour H, Yousefzadeh G, Forood A, Karamouzian M, Shadkam M, Mirzazadeh A. Overweight and obesity prevalence and its predictors in a general population: A community-based study in Kerman, Iran (Kerman coronary artery diseases risk factors studies). ARYA Atheroscler. 2016;12(1):18-27.

16. Tabrizi JS, Sadeghi-Bazargani H, Farahbakhsh M, Nikniaz L, Nikniaz Z. Prevalence and Associated Factors of Overweight or Obesity and Abdominal Obesity in Iranian Population: A Population-based Study of Northwestern Iran. Iran J Public Health. 2018;47(10):1583-1592.

17. Jafari-Adli S, Jouyandeh Z, Qorbani M, Soroush A, Larijani B, Hasani-Ranjbar S. Prevalence of obesity and overweight in adults and children in Iran; a systematic review. J Diabetes Metab Disord. 2014;13(1):121. doi: 10.1186/ s40200-014-0121-2.

18. GBD 2015 Risk Factors Collaborators. Global, regional, and national comparative risk assessment of 79 behavioural, environmental and occupational, and metabolic risks or clusters of risks, 1990-2015: a systematic analysis for the Global Burden of Disease Study 2015. Lancet. 2016;388(10053):1659-1724. doi: 10.1016/S01406736(16)31679-8.
19. Finucane MM, Stevens GA, Cowan M, Danaei G, Lin JK, Paciorek CJ, et al. National, regional, and global trends in body mass index since 1980: Systematic analysis of health examination surveys and epidemiological studies with 960 country-years and 9.1 million participants. Lancet. 2011;377(9765):557-67.

20. Peykari N, Sepanlou S, Djalalinia S, Kasaeian A, Parsaeian M, Ahmadvand A, et al. National and Sub-national Prevalence, Trend, and Burden of Metabolic Risk Factors (MRFs) in Iran: 1990-2013, Study Protocol. Arch Iran Med. 2014;17(1):54-61.

21. Mehdipour P, Navidi I, Parsaeian M, Mohammadi Y, Moradi LM, Rezaei DE, et al. Application of Gaussian Process Regression (GPR) in estimating under-five mortality levels and trends in Iran 1990-2013, study protocol. Arch Iran Med. 2014;17(3):189.

22. Kasaeian A, Eshraghian M, Rahimi FA, Niakan KS, Mohammad K, Farzadfar F. Bayesian autoregressive multilevel modeling of burden of diseases, injuries and risk factors in Iran 1990-2013. Arch Iran Med. 2014;17(1):22-7.

23. Parsaeian M, Farzadfar F, Zeraati H, Mahmoudi M, Rahimighazikalayeh G, Navidi I, et al. Application of spatio-temporal model to estimate burden of diseases, injuries and risk factors in Iran 1990-2013. Arch Iran Med. 2014;17(1):28-33

24. Ng M, Fleming T, Robinson M, Thomson B, Graetz N, Margono C, et al. Global, regional, and national prevalence of overweight and obesity in children and adults during 1980-2013: a systematic analysis for the Global Burden of Disease Study 2013. Lancet. 2014;384:766-81. doi: 10.1016/ S0140-6736(14)60460-8.

25. Stevens GA, Singh GM, Lu Y, Danaei G, Lin JK, Finucane MM, et al. National, regional, and global trends in adult overweight and obesity prevalences. Popul Health Metr. 2012;10(1):22. doi: 10.1186/1478-7954-10-22.

26. Flegal KM, Kruszon-Moran D, Carroll MD, Fryar CD, Ogden CL. Trends in obesity among adults in the United States, 2005 to 2014. JAMA. 2016;315(21):2284-91. doi: 10.1001/jama.2016.6458.

27. Djalalinia S, Moghaddam SS, Peykari N, Kasaeian A, Sheidaei A, Mansouri A, et al. Mortality Attributable to Excess Body Mass Index in Iran: Implementation of the Comparative Risk Assessment Methodology. Int J Prev Med. 2015;6:107. doi: 10.4103/2008-7802.169075.

28. Fahed AC, El-Hage-Sleiman AK, Farhat TI, Nemer GM. Diet, genetics, and disease: a focus on the middle East and north Africa region. J Nutr Metab. 2012;2012:109037. doi: 10.1155/2012/109037.

29. Esteghamati A, Khalilzadeh O, Rashidi A, Kamgar M, Meysamie A, Abbasi M. Physical Activity in Iran: Results of the Third National Surveillance of Risk Factors of NonCommunicable Diseases (SuRFNCD-2007). J Phys Act Health. 2011;8(1):27-35. doi: 10.1123/jpah.8.1.27.

30. GBD 2015 Obesity Collaborators, Afshin A, Forouzanfar MH, Reitsma MB, Sur P, Estep K, et al. Health effects of overweight and obesity in 195 countries over 25 years. N Engl J Med. 2017;377(1):13-27. doi: 10.1056/ NEJMoa1614362.

31. Kite J, Grunseit A, Bohn-Goldbaum E, Bellew B, Carroll T, Bauman A. A systematic search and review of adult-targeted overweight and obesity prevention mass media campaigns and their evaluation: 2000-2017. J Health Commun. 
2018;23(2):207-232. doi: 10.1080/10810730.2018.1423651.

32. Gadde KM, Martin CK, Berthoud HR, Heymsfield SB. Obesity: Pathophysiology and Management. J Am Coll Cardiol. 2018;71(1):69-84. doi: 10.1016/j.jacc.2017.11.011.

33. Esteghamati A, Etemad K, Koohpayehzadeh J, Abbasi M, Meysamie A, Noshad S, et al. Trends in the prevalence of diabetes and impaired fasting glucose in association with obesity in Iran: 2005-2011. Diabetes Res Clin Pract. 2014;103(2):319-27. doi: 10.1016/j.diabres.2013.12.034.

34. NCD Risk Factor Collaboration (NCD-RisC). Worldwide trends in blood pressure from 1975 to 2015: a pooled analysis of 1479 population-based measurement studies with 19.1 million participants. Lancet. 2017;389(10064):3755. doi: 10.1016/S0140-6736(16)31919-5.

35. Hashemi H LB, Sayari AK, Malekzadeh R, Dinarvand $\mathrm{R}$, Aghajani $\mathrm{M}$, et al. National action plan for noncommunicable diseases prevention and control in Iran; a response to emerging epidemic. J Diabetes Metab Disord. 2017;16:3. doi: 10.1186/s40200-017-0288-4

36. Danial Z, Motamedi MH, Mirhashemi S, Kazemi A, Mirhashemi AH. Ageing in Iran. Lancet. 2014;384(9958):1927. doi: 10.1016/s0140-6736(14)622789.

37. Shepherd RW. No evidence for benefit of medication for obesity. Can Fam Physician. 2017;63(4):276

38. Redsell SA, Edmonds B, Swift JA, Siriwardena AN, Weng S, Nathan D, et al. Systematic review of randomised controlled trials of interventions that aim to reduce the risk, either directly or indirectly, of overweight and obesity in infancy and early childhood. Matern Child Nutr. 2016;12(1):24-38. doi: $10.1111 / \mathrm{mcn} .12184$.

39. Landsberg L, Aronne LJ, Beilin LJ, Burke V, Igel LI, LloydJones D, et al. Obesity-related hypertension: pathogenesis, cardiovascular risk, and treatment. J Clin Hypertens (Greenwich). 2013;15(1):14-33. doi: 10.1111/jch.12049

40. Hall ME, do Carmo JM, da Silva AA, Juncos LA, Wang Z, Hall JE. Obesity, hypertension, and chronic kidney disease. Int J Nephrol Renovasc Dis. 2014;7:75-88. doi: 10.2147/ IJNRD.S39739.

41. Owen-Smith A, Coast J, Donovan JL. Self-responsibility, rationing and treatment decision making - managing moral narratives alongside fiscal reality in the obesity surgery clinic. Health Expect. 2018;21(3):606-614. doi: 10.1111/ hex.12651.

42. Moores CJ, Bell LK, Miller J, Damarell RA, Matwiejczyk L, Miller MD. A systematic review of community-based interventions for the treatment of adolescents with overweight and obesity. Obes Rev. 2018;19(5):698-715. doi: 10.1111/obr.12660.

43. Salgado-Peralvo AO, Mateos-Moreno MV, Arriba-Fuente L, Garcia-Sanchez A, Salgado-Garcia A, Peralvo-Garcia V et al. Bariatric surgery as a risk factor in the development of dental caries: a systematic review. Public Health. 2018;155:26-34. doi: 10.1016/j.puhe.2017.11.013.

44. Woodruff RC, Raskind IG, Harris DM, Gazmararian JA, Kramer M, Haardorfer R et al. The dietary impact of introducing new retailers of fruits and vegetables into a community: results from a systematic review. Public Health Nutr. 2018;21(5):981-991. doi: 10.1017/ S1368980017003226.

45. Burgermaster M, Contento I, Koch P, Mamykina L. Behavior change is not one size fits all: psychosocial phenotypes of childhood obesity prevention intervention participants. Transl Behav Med. 2018;8(5):799-807. doi: 10.1093/tbm/ ibx029. 